\title{
Mathematical Modelling of Glucose-Insulin System and Test of Abnormalities of Type 2 Diabetic Patients
}

\author{
Wellars Banzi $\left(\mathbb{D},{ }^{1}\right.$ Immaculate Kambutse ${ }^{D},{ }^{2}$ Vincent Dusabejambo $\left(\mathbb{D},{ }^{2}\right.$ \\ Eric Rutaganda $(\mathbb{D}),{ }^{2}$ Froduald Minani $\left(\mathbb{D},{ }^{1}\right.$ Japhet Niyobuhungiro $(\mathbb{D})$, \\ Lydie Mpinganzima $\mathbb{D}^{1}{ }^{1}$ and Jean Marie Ntaganda $\mathbb{D}^{1}$ \\ ${ }^{1}$ University of Rwanda, College of Science and Technology, School of Science, Department of Mathematics, Kigali, Rwanda \\ ${ }^{2}$ University of Rwanda, College of Medicine and Health Sciences, School of Medicine and Pharmacy, Kigali, Rwanda \\ Correspondence should be addressed to Jean Marie Ntaganda; jmnta@yahoo.fr
}

Received 8 October 2020; Accepted 9 January 2021; Published 3 February 2021

Academic Editor: Marianna A. Shubov

Copyright ( 2021 Wellars Banzi et al. This is an open access article distributed under the Creative Commons Attribution License, which permits unrestricted use, distribution, and reproduction in any medium, provided the original work is properly cited.

\begin{abstract}
This paper presents a mathematical model of glucose-insulin dynamics which is specific for type 2 diabetic patients. The general modelling is obtained by simplification of a global compartmental model by John Thomas Sorensen. The model parameters are estimated using nonlinear optimization and data collected in Rwanda for type 2 diabetic patients. In order to identify and evaluate possible abnormalities of type 2 diabetic patients, the Sampling Importance Resampling (SIR) particle filtering algorithm is used and implemented through discretization of the developed mathematical model. This process is done by clamping insulin and glucose concentrations at around clinical trial values as proposed by Defronzo. Furthermore, for detecting potential abnormalities in type 2 diabetic patients, we compare our results with results obtained from a simulation of the mathematical model for healthy subjects. The proposed mathematical model allows further investigation of the dynamic behavior of glucose, insulin, glucagon, stored insulin, and labile insulin in different organs for type 2 diabetic patients.
\end{abstract}

\section{Introduction}

Diabetes mellitus is one of the metabolic diseases that affect a large number of people in the world. It is characterized by chronic hyperglycemia resulting from defects in insulin secretion, insulin action, or both [1]. Since antiquity chronic disease is recognized by researchers, in about $1550 \mathrm{BC}$, frequent urination and excessive drinking were known on Egyptian papyrus [2]. Four main groups of subdivision were suggested by the World Health Organization (WHO) in 1999 [3]: (1) Young children are affected by type 1, which is due to failure to produce enough insulin. (2) Lack of physical activity and excessive body cause type 2 and either the cells of patients who suffer from type 2 fail to respond to insulin properly or the pancreas does not produce enough insulin. (3) Pregnant women without any antecedent diabetes can suffer from gestational diabetes due to high blood sugar levels but this diabetes similar to type 2 usually disappears after the woman gives birth to her new baby. (4) The causes of specific types include genetic defects in $\beta$-cells function and in insulin action. Among other causes of these specific types are genetic syndromes associated with diabetes and diabetes secondary to other conditions such as pancreatitis and cystic fibrosis.

American Diabetes Association [4] argued the difference between two main types of diabetes, that is, type 1 and type 2 . Indeed, the destruction of pancreatic $\beta$-cells due to an autoimmune process is the cause of type 1 , while the failure of $\beta$-cells in secretion of adequate insulin and insulin resistance in muscles and adipose tissues lead to type 2 , which is a progressive disease. As metabolic illness, type 1 or type 2 affects internal organs of the body. These include pancreas, heart, liver, and lungs. Type 1 or type 2 causes kidney to suffer from nephropathy when the small blood vessels are damaged. If type 1 or type 2 is not well monitored, it has a negative impact on limbs. Diabetes mellitus does not affect only the internal organs of human body, but it may also affect the senses. The most normally targeted is the sense of sight. Furthermore, the blindness is caused by seriousness of the retina damage (retinopathy). Long suffering of the human body 
thanks to diabetes mellitus can also have harmful effects on other organs when nerves are damaged (neuropathy) [3]. Some cardiovascular diseases such as stroke, heart disease, or heart attack result in abnormalities associated with diabetes mellitus. Even if the level of blood sugar can reach $175 \mathrm{mg} / \mathrm{dl}$ after eating, this level decreases after a short time if a person is normal and does not suffer from diabetes. This is not the case for a diabetic patient, since the blood sugar level is often maintained at 180 after eating. The dysfunction of $\beta$-cells enables impaired glucose tolerance and impaired fasting glucose, that is, when there is no caloric intake for at least 8 hours. The consequence of this dysfunction is type 2 diabetes and failure of the cells to respond to glucagon, which prevents blood glucose levels from dropping too low.

Often the statistics based on death certificates show that individuals do not die only of cardiovascular and renal disease but also diabetes and effects uniquely related to it are the main cause of death in the world [5]. This is global challenge to estimate deaths attributable to diabetes. In 2007, International Diabetes Federation argued in the report that more than 300 million people in the world are affected by diabetes [6]. The prediction of this disease show that it can affect 380 million by 2025 [7]. It is estimated that 14.2 million adults suffer from diabetes in Sub-Saharan Africa. The causes of this big number are limited health systems and lack of skilled healthcare providers [8]. Although most countries of this region do not have reliable data, it is expected that diabetes will affect 34.2 million adults by the year 2040 [9]. In addition, among identified cases of $90-95 \%$ in this region are type 2 diabetic patients [10]. In Rwanda, an approximate of 194,300 diabetic cases were diagnosed in adults in 2015 and the country registered 5000 diabetes-related deaths $[11,12]$. It is known that there is a direct relationship, even a correlation, between overweight population and the increase of metabolic diseases. In Rwanda, the level of treatment of type 2 diabetes as endocrine disorders is highly appreciated but it is costing a lot of money [13]. Between October 1, 2006, and September 30, 2014, Tapela et al. conducted a study on noncommunicable diseases (NCDs) in rural districts in Rwanda [14]. The sample size was 544 patients and the outcomes of their study show that the majority suffered from type 2 diabetes and 10.3\% were the median baseline glycated hemoglobin $(\mathrm{HbA} 1 \mathrm{c})$. Despite all these difficulties, efforts have been made to combat diabetes mellitus [15-17]. Since 2006, support came from World Diabetes Foundation to Rwandan Diabetes Association to fund the training healthcare providers in 15 Rwandan districts. In Rwanda, the world diabetes day represents a key initiative to raise awareness on diabetes mellitus, where public speaking, sensitization walk, mass screening of diabetes with involvement of Rwandan diabetic association, and Ministry of Health through RBC (Rwanda Biomedical Center) coordinate all activities. Although Rwanda is one of the few countries in Sub-Saharan Africa which have the strategic plan to treat NCDs; chronic disease management remains a major challenge $[11,18]$. The role of mathematics in controlling diabetes mellitus is materialized by several mathematical models of glucose and insulin. Some of them deal with glucose regulation in healthy human subjects [19-21] and others focus on therapy [22]. Among all those, the most popular approach is compartmental modelling. In 1981, Bergman et al. [23] developed the minimal compartmental model of diabetes dynamics. This mathematical model has been used in diabetic studies [24]. However, more complicated mathematical models have been developed by different authors: Cobelli and Mari [25], Sorensen [26], and Hovorka et al. [27]. The mathematical modelling of glucose dynamics and metabolism in human body is very essential. Indeed, the adjustment and modification of this mathematical model lead to study the physiological behavior of type 2 diabetic patients. This mechanism is made by developing a mathematical model with adapted parameters. The same approach has been used by Vahidi [28]. However, type 2 diabetes modelling is not as simple, since there are several abnormalities associated with the disease in different organs, which in turn are associated with the deterioration of glucose homeostasis. Glucose metabolic rates in the related organs are affected by these abnormalities. Moreover, these abnormalities also target the secretion rate of insulin in the pancreas and other regulatory hormones in terms of their glucose secretion rates. The objective of this work is to test abnormalities of type 2 diabetic patients in Rwanda using adapted mathematical model of glucose-insulin system presented in [29]. The model equations are nonlinear, so that their resolution requires the use of sophisticated computational tools. In Eleni and Smyth's work [30], the list of techniques which are nonlinear has been proposed to be applied in civil engineering. These include the Unscented Kalman Filter (UKF), Least Squares Estimation (LSE), the Extended Kalman Filter (EKF), and Sequential Monte Carlo Methods (Particle Filters). Wu and Smyth [31] argued that the most used technique in state estimating and in identifying parameters is $\mathrm{UKF}$ rather than EKF, since UKF is more robust and uses higher degree of freedom systems to measure the noise levels. In addition, Kalman filters play a crucial role in several fields of science, particularly in structural health monitoring [32]. This work deals with the particle filter algorithm to test abnormalities of glucose-insulin system. Moreover, the numerical simulations are carried out using Sampling Importance Resampling (SIR) algorithm for the particle filter [33-36].

\section{Materials and Methods}

2.1. Data Sources. Physicians consented purposefully patients known with type 2 diabetes coming for regular follow-up in public hospital in Kigali, Rwanda. The protocols were approved by the Ethical Committee of College of Medicine and Health Sciences (CMHS) Institutional Review Board (IRB) at the University of Rwanda. Selected patients were adult patients on treatment (oral medications, insulinotherapy, or combined oral medications and insulinotherapy). After consent, the selected patients were asked to get fasting blood sugars at home and a sample was taken at least 8 hours after the last meal to determine the fasting glucose blood levels. A blood sample is taken and different tests are performed to determine the individual's blood glucose levels. Patients with end-stage renal disease were excluded, since their glycemia is pathophysiologically normal despite being diabetic. Confused patients and patients with inability to consent for this study were excluded. $\mathrm{HbA} 1 \mathrm{C}$ in some of patients who had clear indication were done in laboratories of the same institution. Physician recorded them on different days and reported them to the research team as a 
summarized progressive report of records. The data are collected for 103 patients (51 males and 52 females) but on daily basis, 10 patients have been followed up where needed and medical measures were recorded every morning for a period of one month. All HbA1C were reported.

2.2. Model Equations. For a healthy human being, a global mathematical model developed by Sorensen [26] is widely used. It is a compartmental model with 6 compartments and a system of 22 ordinary differential equations. The complexity of this mathematical model in terms of resolution and numerical simulations motivated us to develop a simplified mathematical model for type 2 diabetic patients [29]. The proposed mathematical model constitutes three main concentrations in the human body: glucose, insulin, and glucagon. We mainly consider four compartments: heart and lungs, liver, tissues, and pancreas. The tissues compartment constitutes a set of brain, kidney, gut, and periphery. To test, we adapt this model by introducing two additional compartments for the insulin related to a small labile insulin and to a large insulin storage [37]. Therefore, the mathematical model is comprised of four subsystems: glucose, insulin, glucagon, and pancreas. Table 1 shows the nomenclature of variables and parameters, while Table 2 presents parameters to be estimated.

In Figure 1, we give a schematic representation of the model for a diabetic patient.

The general form of the mass balance equation for glucose, insulin, and glucagon submodels is as follows:

$$
V \frac{M}{\mathrm{~d} t}=Q\left(M_{\text {in }}-M_{\text {out }}\right)+R_{p}-R_{c},
$$

where $V$ is the volume of compartment, $M$ denotes the concentration of either insulin, glucose, or glucagon, $t$ is the time, $Q$ is the blood flow rate, and $R_{p}$ and $R_{c}$ are the metabolic production and consumption rates of the material balance substance, respectively. The adapted and simplified mathematical model of glucose and insulin to test abnormalities of type 2 diabetic patients in Rwanda is defined by three parts of mathematical system. The first system is the six following ordinary differential equations:

$$
\left\{\begin{array}{l}
\frac{\mathrm{d} G_{H}(t)}{\mathrm{d} t}=\frac{1}{V_{H}^{G}}\left[Q_{L}^{G} G_{L}(t)+\gamma_{T}^{G}\left(G_{T}(t)\right)^{\alpha}-Q_{H}^{G} G_{H}(t)-R_{H}^{G}\right] \\
\frac{\mathrm{d} G_{L}(t)}{\mathrm{d} t}=\frac{1}{V_{L}^{G}}\left[Q_{A}^{G} G_{H}(t)-Q_{L}^{G} G_{L}(t)+R_{L}^{G}\right] \\
\frac{\mathrm{d} G_{T}(t)}{\mathrm{d} t}=\frac{1}{V_{T}^{G}}\left[Q_{P}^{G}\left(G_{H}(t)-\gamma_{T}^{G} G_{T}(t)\right)-R_{T}^{G}\right] \\
\frac{\mathrm{d} I_{H}(t)}{\mathrm{d} t}=\frac{1}{V_{H}^{I}}\left[Q_{L}^{I} I_{L}(t)+\gamma_{T}^{I}\left(I_{T}(t)\right)^{\beta}-Q_{H}^{I} I_{H}(t)\right] \\
\frac{\mathrm{d} I_{L}(t)}{\mathrm{d} t}=\frac{1}{V_{L}^{I}}\left[Q_{A}^{I} I_{H}(t)-Q_{L}^{I} I_{L}(t)+R_{P I R}-R_{L}^{I}\right] \\
\frac{\mathrm{d} I_{T}(t)}{\mathrm{d} t}=\frac{1}{V_{T}^{I}}\left[Q_{P}^{I}\left(I_{H}(t)-\gamma_{T}^{I} I_{T}(t)\right)-R_{T}^{I}\right]
\end{array}\right.
$$

where the first three equations consist of glucose submodel, while the last three are insulin submodel.

The dynamics of glucagon have effects on the metabolic rates of glucose. One compartment is enough to model effects of glucagon hormone. The second part of mathematical system to test abnormalities of type 2 diabetic patients in Rwanda is the following equation adapted from Sorensen [26] to justify the role of glucagon and to present glucagon submodel:

$$
\frac{\mathrm{d} \Gamma(t)}{\mathrm{d} t}=\frac{1}{V^{\Gamma}}\left[\eta G_{H} I_{H}-R^{\Gamma} \Gamma\right],
$$

where $\eta$ is a multiplicative constant for plasma glucagon clearance rate and $R^{\Gamma}$ denotes the rate for pancreatic glucagon release. The third model system and the last to test abnormalities of type 2 diabetic patients are constructed by taking into account the pancreas compartment updated to non-insulin-dependent diabetes mellitus conditions. Furthermore, the change in blood glucose concentration allows pancreatic insulin to release. Landahl and Grodsky [37] developed mathematical model of this pancreatic mechanism using two compartments: a small labile insulin compartment and a large insulin storage compartment. This mathematical model shows a biphasic insulin release response to glucose stimulation [38] for type 2 diabetic patients. Taking into account the fact that insulin is secreted from $\beta$-cells in the pancreas in a complex mechanism and considering mathematical model developed by in Landahl and Grodsky [37], we propose the following mass balance equations of pancreas submodel:

$$
\left\{\begin{array}{l}
\frac{\mathrm{d} I_{s}(t)}{\mathrm{d} t}=-p_{s} I_{s}(t)+\left(I_{b}(t)\right)^{\sigma} G_{H}(t), \\
\frac{\mathrm{d} I_{s}(t)}{\mathrm{d} t}=-p_{b} I_{b}(t)+\left(I_{s}(t)\right)^{\delta} G_{H}(t) .
\end{array}\right.
$$

Finally, the mathematical model to test abnormalities of type 2 diabetic patients in Rwanda is a system of 9 ordinary differential equations (2)-(4) along with the nomenclature shown in Tables 1 and 2.

\section{Model Parameters}

The parameters for the patient model have been adjusted using the blood glucose clinical data collected at three different hospitals in Kigali, Rwanda. Notice that a large sample size is not mandatory to determine parameters in compartmental theory such as blood insulin and/or glucose. In this paper, a sample of 10 patients are followed up on daily basis and have been considered and the fasting blood sugar levels were recorded every morning for a period of one month. Using the average taken as daily blood sugar level, the blood insulin data are obtained using Bergman's insulin minimal model [23]:

$$
\begin{cases}\frac{\mathrm{d} I(t)}{\mathrm{d} t}=k_{1}\left(G_{t}-G^{*}\right) t-k_{2} I(t), & \text { if } G_{t}>G^{*} \\ \frac{\mathrm{d} I_{s}(t)}{\mathrm{d} t}=-k_{2} I(t), & \text { otherwise, }\end{cases}
$$


TABLE 1: Nomenclature of mathematical model.

\begin{tabular}{lc}
\hline Variable & Description \\
\hline$V$ & Volume (dl) \\
$G$ & Glucose concentration (mg/dl) \\
$I$ & Insulin concentration (mU/l) \\
$Q$ & Vascular blood flow rate (dl/min) \\
\hline Subscript & Description \\
\hline$H$ & Heart and lungs \\
$L$ & Liver \\
$T$ & Tissues \\
$A$ & Hepatic artery \\
PIR & Peripheral insulin release \\
$s$ & Stored insulin \\
$b$ & Labile insulin \\
\hline Subscript & Description \\
\hline$G$ & Glucose \\
$I$ & Insulin \\
$\Gamma$ & Glucagon \\
\hline
\end{tabular}

TABLE 2: Nomenclature of mathematical model parameters to be estimated.

\begin{tabular}{lc}
\hline Constant to be estimated & Description \\
\hline$R$ & Metabolic source or sink rate $(\mathrm{mg} / \mathrm{min}$ or $\mathrm{mU} / \mathrm{min})$ \\
$\gamma$ & Vascular blood flow rate $(\mathrm{dl} / \mathrm{min})$ \\
$p$ & Parameter \\
$\alpha$ & Parameter \\
$\delta$ & Parameter \\
$\sigma$ & Parameter \\
$\beta$ & Parameter \\
\hline
\end{tabular}

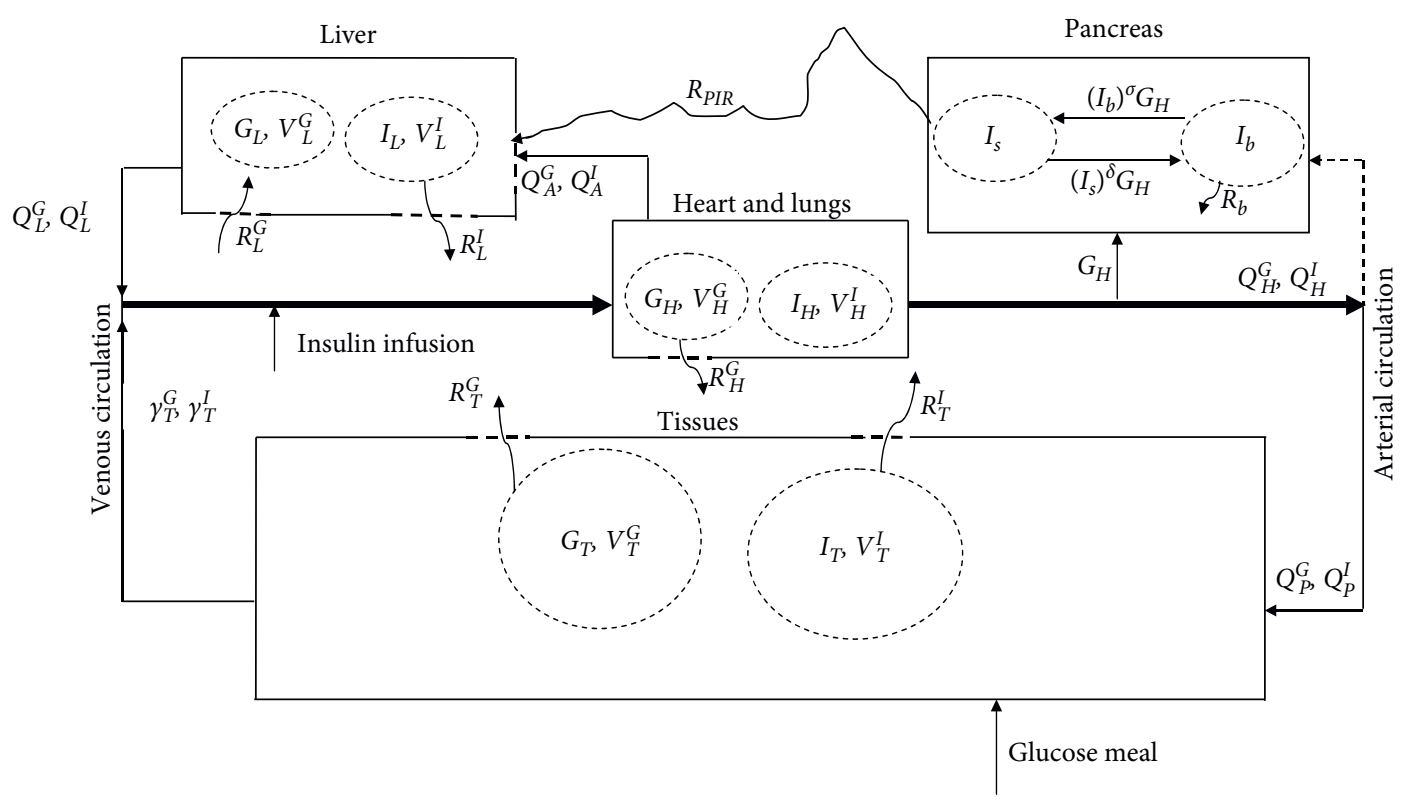

FIGURE 1: Schematic representation of the model for a diabetic patient. We have three main physiological compartments, namely, heart, liver, tissues, and pancreas. The tissues compartment is introduced as one compartment that contains four additional compartments (brain, kidney, gut, and periphery, i.e., skeletal muscle and adipose tissue, vascular-lymphatic system, skin, bones, and peripheral nervous system). The direction of blood flow is represented by the arrows which connect the physiological compartments. For notational purposes, we use subscripts in order to distinguish the physiological compartments. They indicate fluid spaces within compartments. 
with initial condition $I(0)=I_{0}$, where $k_{1}$ and $k_{2}$ are parameters of mathematical model, $G_{t}$ is the value of glucose at a given time $t$, and $G^{*}$ denotes the threshold for blood glucose concentration. Solving equation (5), we get

$$
\begin{cases}\frac{\mathrm{d} I(t)}{\mathrm{d} t}=e^{-k_{2} t}\left[\frac{k_{1}\left(G_{t}-G^{*}\right)}{k_{2}}\left(1+\left(t-\frac{1}{k_{2}}\right) e^{k_{2} t}+I_{0}\right)\right], & \text { if } G_{t}>G^{*}, \\ \frac{\mathrm{d} I_{s}(t)}{\mathrm{d} t}=I_{0} e^{-k_{2} t}, & \text { otherwise. }\end{cases}
$$

In numerical test, we consider the values of $k_{1}, k_{2}$, and $G^{*}$ as $0.0055,0.29$ [23], and 92.5 [26], respectively.

The parameter values used for type 2 diabetes can be found in the literature $[26,29]$, and they are summarized in Table 3.

For identification of the parameters of mathematical model (2)-(4), we need to solve a nonlinear optimization problem formulated below. Let

$$
\begin{aligned}
X & =\left(G_{H}, G_{L}, G_{T}, I_{H}, I_{L}, I_{T}, \Gamma, I_{s}, I_{b}\right)^{t}, \\
f_{1}(t) & =\frac{1}{V_{H}^{G}}\left[Q_{L}^{G} G_{L}(t)+\gamma_{T}^{G}\left(G_{T}(t)\right)^{\alpha}-Q_{H}^{G} G_{H}(t)-R_{H}^{G}\right], \\
f_{2}(t) & =\frac{1}{V_{L}^{G}}\left[Q_{A}^{G} G_{H}(t)-Q_{L}^{G} G_{L}(t)+R_{L}^{G}\right], \\
f_{3}(t) & =\frac{1}{V_{T}^{G}}\left[Q_{P}^{G}\left(G_{H}(t)-\gamma_{T}^{G} G_{T}(t)\right)-R_{T}^{G}\right], \\
f_{4}(t) & =\frac{1}{V_{H}^{I}}\left[Q_{L}^{I} I_{L}(t)+\gamma_{T}^{I}\left(I_{T}(t)\right)^{\beta}-Q_{H}^{I} I_{H}(t)\right], \\
f_{5}(t) & =\frac{1}{V_{L}^{I}}\left[Q_{A}^{I} I_{H}(t)-Q_{L}^{I} I_{L}(t)+R_{P I R}-R_{L}^{I}\right], \\
f_{6}(t) & =\frac{1}{V_{T}^{I}}\left[Q_{P}^{I}\left(I_{H}(t)-\gamma_{T}^{I} I_{T}(t)\right)-R_{T}^{I}\right], \\
f_{7}(t) & =\frac{1}{V^{\Gamma}}\left[\eta G_{H} I_{H}-R^{\Gamma} \Gamma\right], \\
f_{8}(t) & =-p_{s} I_{s}(t)+\left(I_{b}(t)\right)^{\sigma} G_{H}(t), \\
f_{9}(t) & =-p_{b} I_{b}(t)+\left(I_{s}(t)\right)^{\delta} G_{H}(t) .
\end{aligned}
$$

It follows that the mathematical model can be rewritten as

$$
\dot{X}(t)=F(X(t), \mu)
$$

where

$$
F=\left(f_{1}, f_{2}, f_{3}, f_{4}, f_{5}, f_{6}, f_{7}, f_{8}, f_{9}\right)^{\prime} \text {, }
$$

and $\mu$ is a column vector of the parameters, that is,

$$
\mu=\left(\gamma_{H}^{G}, \alpha, R_{H}^{G}, R_{L}^{G}, \gamma_{H}^{I}, \beta, R_{L}^{I}, R_{T}^{G}, R_{T}^{I}, \eta, p_{s}, \sigma, p_{b}, \delta\right)^{\prime} .
$$

Using this setting, the optimization problem is

$$
\min _{\mu} \sum_{i=1}^{n}\left(q_{G}\left(G_{T}^{i}(t)-G_{T}^{\mathrm{obs}}\right)+q_{I}\left(I_{T}^{i}(t)-I_{T}^{\mathrm{obs}}\right)\right),
$$

under the constraints in (8), where $q_{G}$ and $q_{I}$ are the weights and $n$ is the clinical sample size. The superscript "obs" refers to the observed clinical measurements of the concentrations of glucose and insulin. We perform a dynamic simulation of a healthy human being using the mathematical model (2). On the other hand, the simulation results of type 2 diabetic patients are obtained by considering the mathematical model (2)-(4). The clinical data for healthy human being are obtained from the numerical resolution of global model developed in [35] using MATLAB solvers of ordinary differential equations such as ode 45 and the optimization is carried out using built-in functions such as fmincon. Figures 2 and 3 show the overall trend of concentrations of glucose and insulin compared with clinical data for healthy human body and type 2 diabetic patients, respectively. The estimated parameters of the mathematical model are shown in Table 4.

\section{Test of Abnormalities of Type 2 Diabetic Patients}

The physiology of glucose-insulin system provides insights on abnormalities associated with the patients. Therefore, since the mathematical model is a representation of this physiological behavior, we can use it to test abnormal functionality in the liver, tissues, and other organs. For diabetic patients, high levels of concentrations in blood glucose in the body are an indication of the abnormal functionality. Therefore, if the rates of glucose and insulin in different organs are compared with their corresponding rate of a healthy body, we can have insights into the level of organ malfunction. To perform the comparison, the model equations (2) are considered as the dynamic model of a healthy subject. This model is completed by equations (3) and (4) in order to get the dynamic model for patients who suffer from type 2 diabetes. The basal conditions of concentrations of glucose and insulin in the tissue for diabetic and healthy subjects are reported in Nagasaka et al.'s work [39] but we use those given in Vahidi's work [28]. They are $117 \pm 7 \mathrm{mg} / \mathrm{dl}$ and $4.5 \pm 0.4 \mu \mathrm{U} / \mathrm{ml}$ for the diabetic group and $85 \pm 8 \mathrm{mg} / \mathrm{dl}$ and $5.1 \pm 0.3 \mu \mathrm{U} / \mathrm{ml}$ for healthy subjects. Defronzo et al. [40] proposed two original tests: the first requires maintaining a high insulin level by perfusion or infusion with insulin (euglycemic hyperinsulinemic clamp), while the second requires maintaining a high blood sugar level by perfusion or infusion with glucose (hyperglycemic clamp). Using the first test, the experimental procedure of continuous insulin infusion leads to raising the plasma insulin concentration at around $10 \mu \mathrm{U} / \mathrm{ml}$, while the plasma glucose concentration remains at basal levels by glucose injection based on negative feedback principle [40]. Therefore, the effect of insulin sensitivity in human body is justified by the measurement glucose infusion rate. The second test is performed by raising and maintaining plasma glucose concentration at $125 \mathrm{mg} / \mathrm{dl}$ which is above basal 
TABle 3: Parameters used from literature.

\begin{tabular}{lc}
\hline Parameter & Value \\
\hline$V_{H}^{G}$ & 3.5 \\
$V_{L}^{G}$ & 25.1 \\
$V_{T}^{G}$ & 9.5 \\
$V_{H}^{I}$ & 0.99 \\
$V_{L}^{I}$ & 1.14 \\
$V_{T}^{I}$ & 0.73 \\
$V^{\Gamma}$ & 6.74 \\
$R^{\Gamma}$ & 9.1 \\
$R_{P I R}$ & 27.8553 \\
$Q_{L}^{G}$ & 12.6 \\
$Q_{H}^{G}$ & 43.7 \\
$Q_{A}^{G}$ & 2.5 \\
$Q_{P}^{G}$ & 12.6 \\
$Q_{L}^{I}$ & 0.9 \\
$Q_{H}^{I}$ & 3.12 \\
$Q_{A}^{I}$ & 0.18 \\
$Q_{P}^{I}$ & 1.05 \\
\hline
\end{tabular}

levels. For this second test, a continuous glucose infusion is used but insulin injection is not performed. This process of maintaining amount of glucose infusion rate at steady state of hyperglycemia level requires the measurement made due to glucose metabolized body cells and insulin supplied by the pancreas [40].

In this work, we use two of these clinical trials to evaluate abnormalities of type 2 diabetic patients in Rwanda. As we mentioned above, the abnormalities of type 2 diabetic patients are detected by comparison of patient model results with those of healthy subjects. In order to perform the comparison, we require measuring glucose and insulin from different parts of the body. However, to take blood samples from many internal body organs, for instance, heart and liver, is clinically impossible. Therefore, in order to estimate concentrations of these parameters, we need to rely on measurements from peripheral tissues.

All estimated values are carried out by implementing the Sampling Importance Resampling (SIR) algorithm. This algorithm enables us to estimate glucose and insulin in heart and liver by measuring the noise of glucose and insulin in peripheral tissues as available measured data. For more details about the SIR algorithm, we refer to Andrieu et al. [33], Arulampalam et al. [34], and Doucet et al. [35]. Therefore, in numerical simulations, we consider $M=100$, $T_{\text {max }}=150$ minutes, and $N=1000$ particles and we assume that the states $X_{0}$ and measurement noises have a normal probability distribution with density function $X 0 \sim N$ $(0,5 I 9), V t \sim N(0,0.01 I 9)$, and $E t \sim N(0,0.05 I 2)$, where $N$ means normal distribution and $I$ is identity matrix of order $n$.

The discretization of the mathematical model (2)-(4) is done using fixed-step backward difference approximation by

$$
\frac{\mathrm{d} x}{\mathrm{~d} t} \approx \frac{x_{j}-x_{j-1}}{\Delta t}, \quad 0 \leq t \leq T_{\max }, j=1, \ldots, M,
$$

where $x_{j}=x\left(t_{j}\right)$ and $\Delta t=\left(T_{\max } / M\right)$ with $M$ being total number of discretization points such that $x_{j}=x_{j-1}+\Delta t$.
Hence, we recursively in time estimate the following state dynamic model:

$$
\left\{\begin{array}{l}
X_{t}=F_{t-1}\left(X_{t-1}\right)+V_{t-1} \\
Y_{t}=P X_{t}+E_{t-1},
\end{array}\right.
$$

where $X_{t}$ and $Y_{t}$ are the state vector and the measurement vector, respectively. $V_{t-1}$ and $E_{t-1}$ are the stochastic process and measurement noise, respectively, and they are independent and identically distributed (i.i.d.). The function $F_{t-1}$ refers to the dynamic equations for the process model, while $P$ denotes the matrix from the equations modelling the sensors referred to as the measurement model given as

$$
P=\left(\begin{array}{lllllllll}
0 & 1 & 0 & 0 & 0 & 0 & 0 & 0 & 0 \\
0 & 0 & 0 & 0 & 0 & 1 & 0 & 0 & 0
\end{array}\right) .
$$

We present and discuss the results of the two tests mentioned above, since they are clinical trials performed for both insulin and glucose. The numerical results are given in Figures 4-9.

The numerical results of two above tests are shown in Figures 4 and 5, respectively. Figure 4 indicates that glucose concentrations are maintained at $125 \mathrm{mg} / \mathrm{dl}$ above the basal level for both patients suffering from type 2 diabetes and healthy subjects. Moreover, as can be seen in Figure 6, these tests show that, in heart, glucose concentration is significantly higher in type 2 diabetic patients than in healthy subjects, whereas insulin is lower in the type 2 diabetic patients than in the healthy subjects (see Figure 7). The low insulin justifies why the body is not properly making or does not make the insulin hormone. Therefore, this blood hyperglycemia is an essential characteristic of diabetes in patients. Since the insulin is resistant for type 2 diabetic patients, the pancreas simply is not able to produce enough insulin, which is very important so that human body cells take up glucose and blood sugar or raise blood glucose levels. Figure 5 shows that, during both tests, the concentration of insulin is clamped at $10 \mu \mathrm{U} / \mathrm{ml}$. Consequently, there is no 


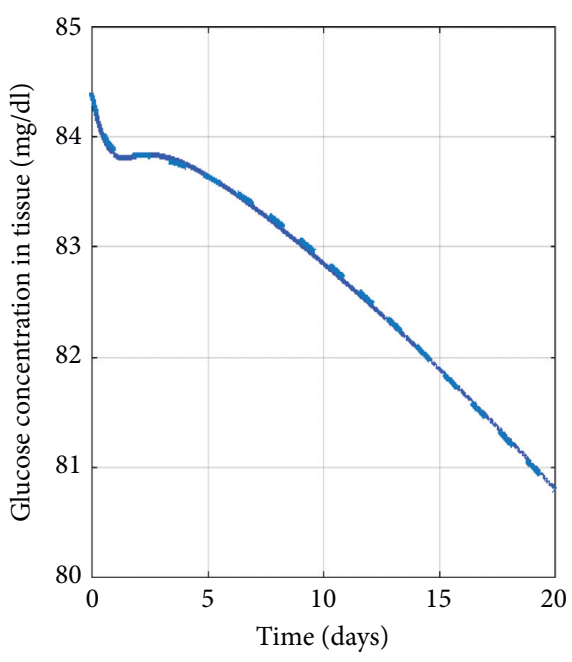

(a)

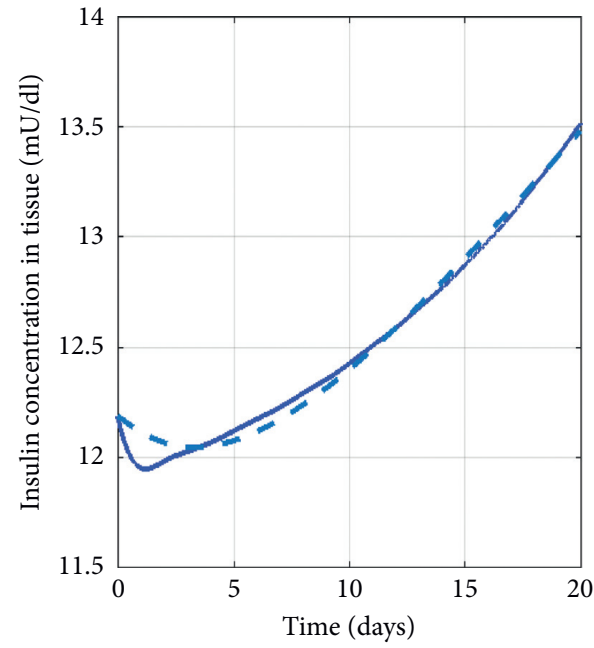

(b)

FIgURE 2: Trends of glucose (a) and insulin (b) concentrations in tissues compared with those of clinical data. The observed data (dashed) and the solution of our mathematical model (solid).

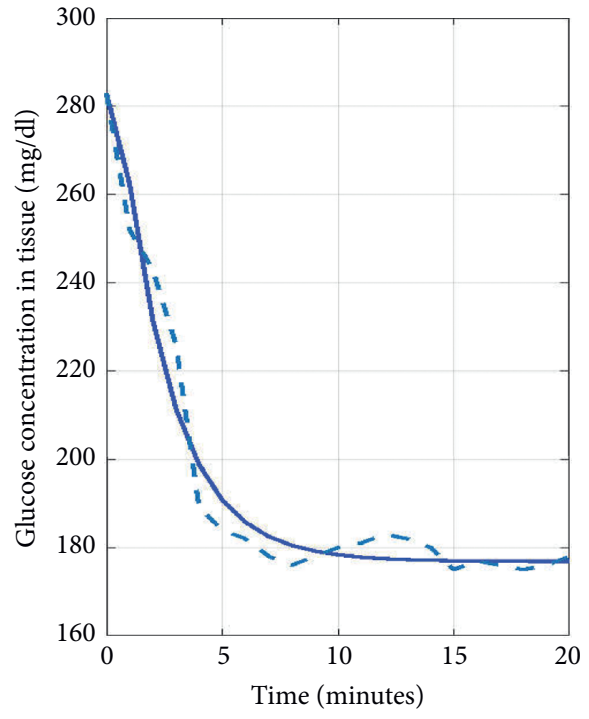

(a)

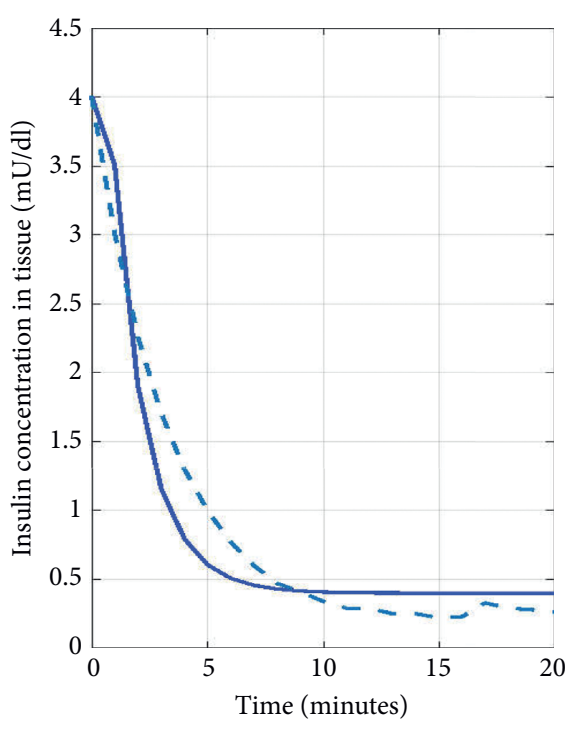

(b)

Figure 3: Trends of glucose (a) and insulin (b) concentrations in tissues compared with those of clinical data. The observed data (dashed) and the solution of our mathematical model (solid).

change of insulin effects as pancreatic hormone on the metabolic rates and the patients' body resistance against insulin remains unchanged. Moreover, the deficiencies in glucose metabolic rate result in glucose resistance in those in liver or tissues. Figure 8 indicates that hepatic glucose is produced at high rate in type 2 diabetic patients compared to one for healthy subjects with normal hepatic glucose. These results are consistent with what happens in glucose metabolism for diabetic patients. Indeed, if type 2 diabetic patients suffer from insulin resistance, the liver often produces glucose when not really needed because insulin is not regulating this sugar. The signal of hormone is not recognized by an insulin resistance so that the glucose does not flow in the blood. That raises blood glucose levels in type 2 diabetic patients compared to the decrease in healthy subjects, as shown in Figure 6. Moreover, the insulin produced in pancreas leads into the liver. Although Figure 9 shows that the insulin in the liver is generally high for the type 2 diabetic patients compared to that of the healthy subjects, it does not allow type 2 diabetic patients to use glucose for energy due to its resistance. Therefore, the blood insulin decreases as shown in Figure 7 and when the insulin is not enough, the body can no longer move glucose from the blood into the cells. This mechanism causes high blood glucose levels. This is illustrated in Figure 6. 
TABLE 4: Estimated model parameters.

\begin{tabular}{lcc}
\hline Parameters & & Value \\
& Healthy & 141.1027 \\
$\gamma_{H}^{G}$ & 17.0590 & 0.8605 \\
$\alpha$ & 0.5257 & 1285.3620 \\
$R_{H}^{G}$ & 1151.6073 & 1256.0874 \\
$R_{L}^{G}$ & 1030.5766 & 5.5286 \\
$\gamma_{H}^{I}$ & 2.5456 & 0.2604 \\
$\beta$ & 1.0636 & 15.4614 \\
$R_{L}^{I}$ & 16.9805 & 85.057 \\
$R_{T}^{G}$ & 122.5738 & 5.5908 \\
$R_{T}^{I}$ & 4.0106 & 0.0550 \\
$\eta$ & - & 1.8813 \\
$p_{s}$ & - & 0.0256 \\
$\sigma$ & - & 7.1415 \\
$p_{b}$ & - & 0.0438 \\
$\delta$ & - & \\
\hline
\end{tabular}

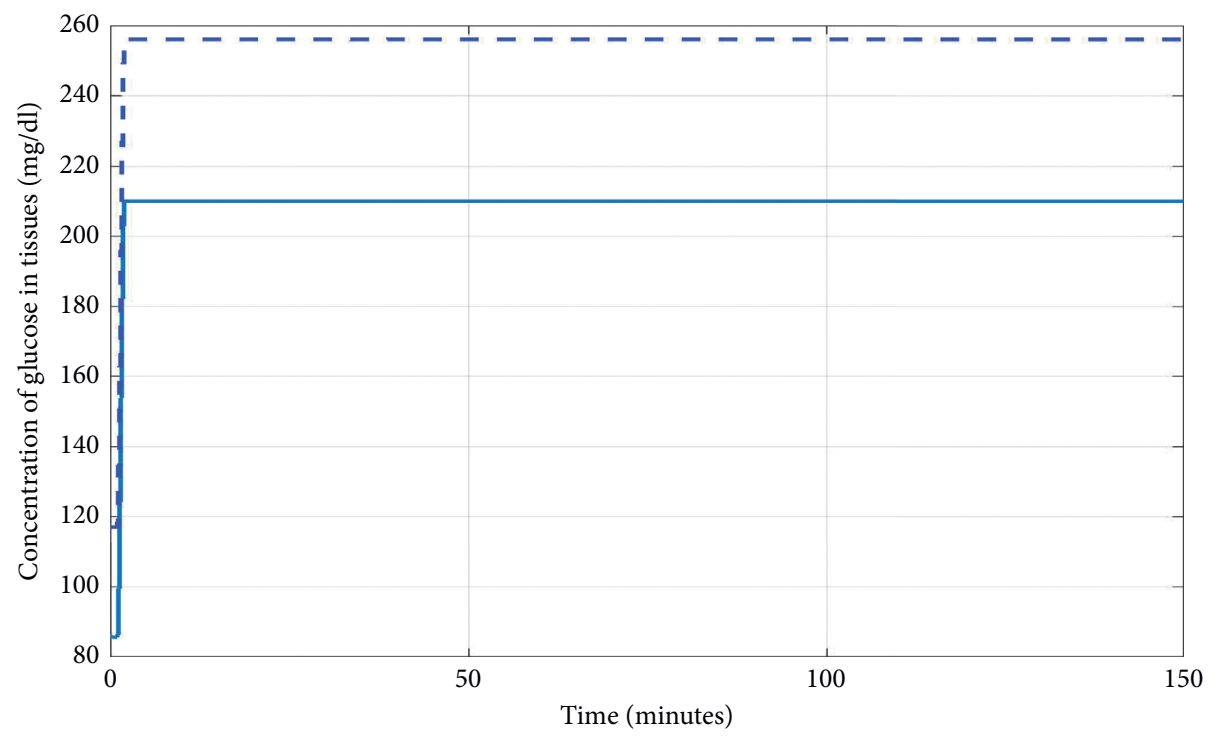

Figure 4: Profile of glucose in tissues during euglycemic hyperinsulinemic clamp test. The dashed line shows the type 2 diabetic patients hyperglycemic clamp test, while the solid line shows the same for healthy subjects.

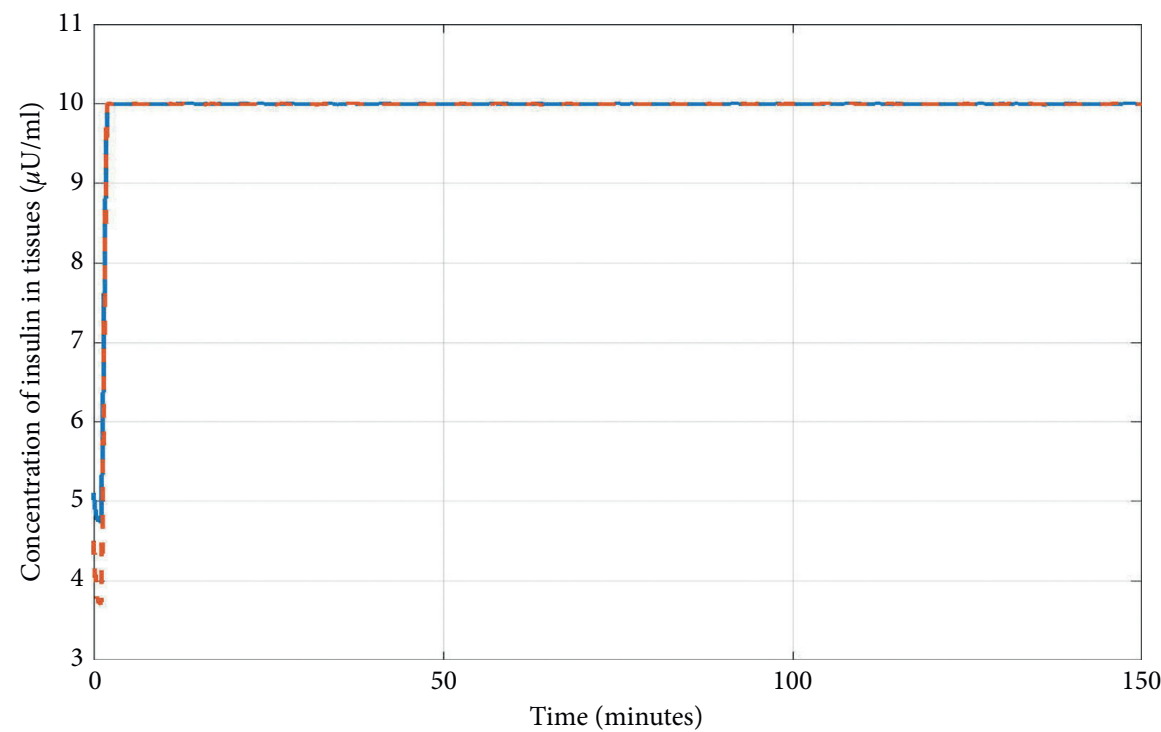

Figure 5: Profile of insulin in tissues during euglycemic hyperinsulinemic clamp test. The dashed line shows the type 2 diabetic patients hyperglycemic clamp test, while the solid line shows the same for healthy subjects. 


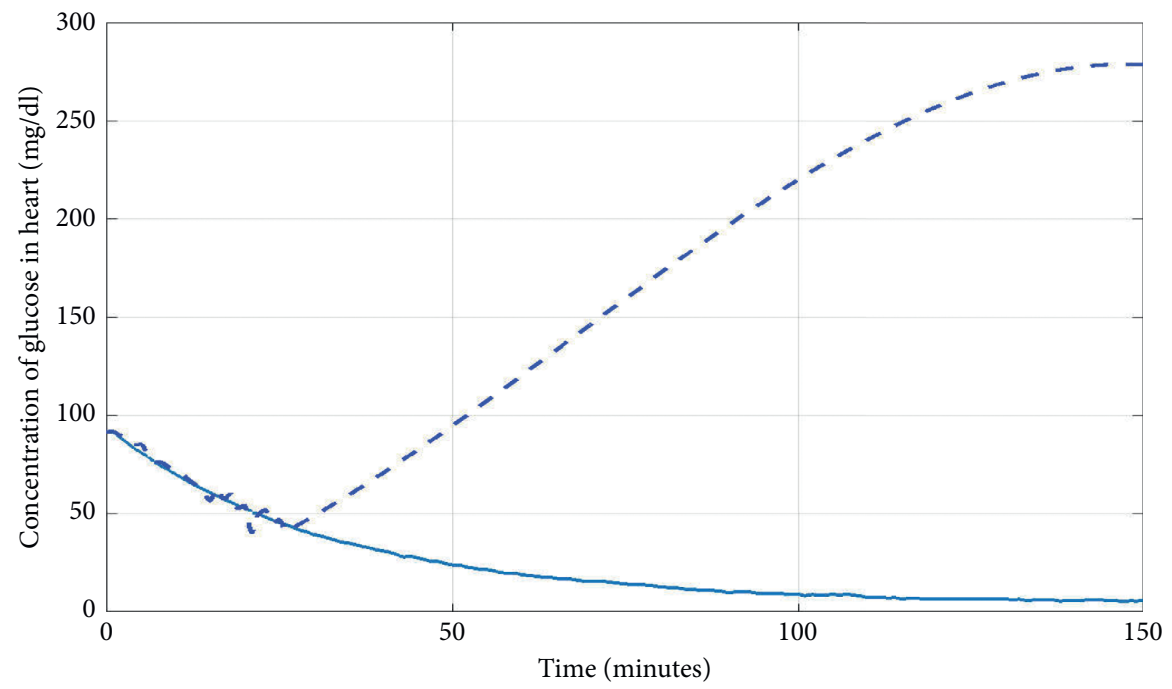

Figure 6: Profile of glucose in heart during euglycemic hyperinsulinemic clamp test. The dashed line shows the type 2 diabetic patients hyperglycemic clamp test, while the solid line shows the same for healthy subjects.

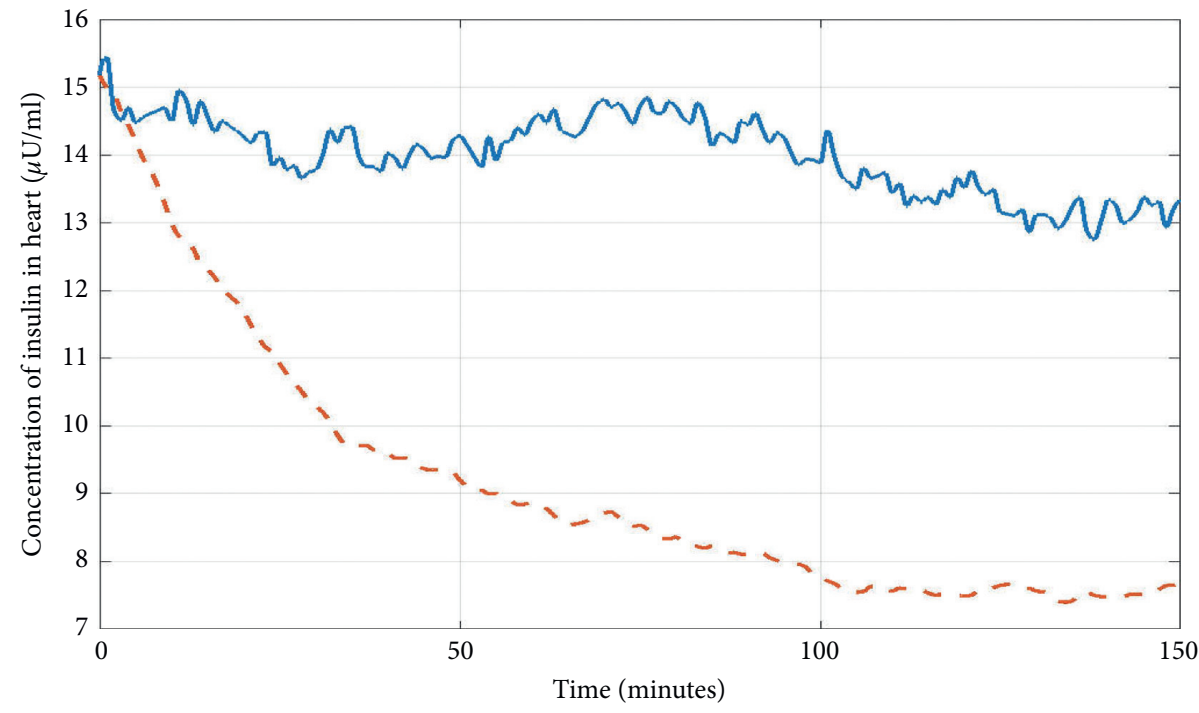

Figure 7: Profile of insulin in heart during euglycemic hyperinsulinemic clamp test. The dashed line shows the type 2 diabetic patients hyperglycemic clamp test, while the solid line shows the same for healthy subjects.

\section{Concluding Remarks}

It is of importance to describe as accurately as possible the physiological behavior of glucose-insulin system by a relatively simple mathematical model. However, the homeostasis of blood glucose in type 2 diabetic patients makes it inherently complex to develop such a model.

In this paper, we have proposed a four-compartmental mathematical model able to describe the variation of glucose-insulin concentrations for type 2 diabetic patients. We used data collected by a regular follow-up of type 2 diabetic patients in public hospitals in Kigali, Rwanda. We have implemented the Sampling Importance Resampling (SIR) particle filtering algorithm using the developed mathematical model in order to detect potential abnormalities in type 2 diabetic patients by comparing numerical results for healthy subjects and type 2 diabetic patients. The results of our simulations are found to be clinically consistent with physiological responses. The proposed mathematical model can also be used by physiologists and other experts in medicine for monitoring glucose-insulin system. 


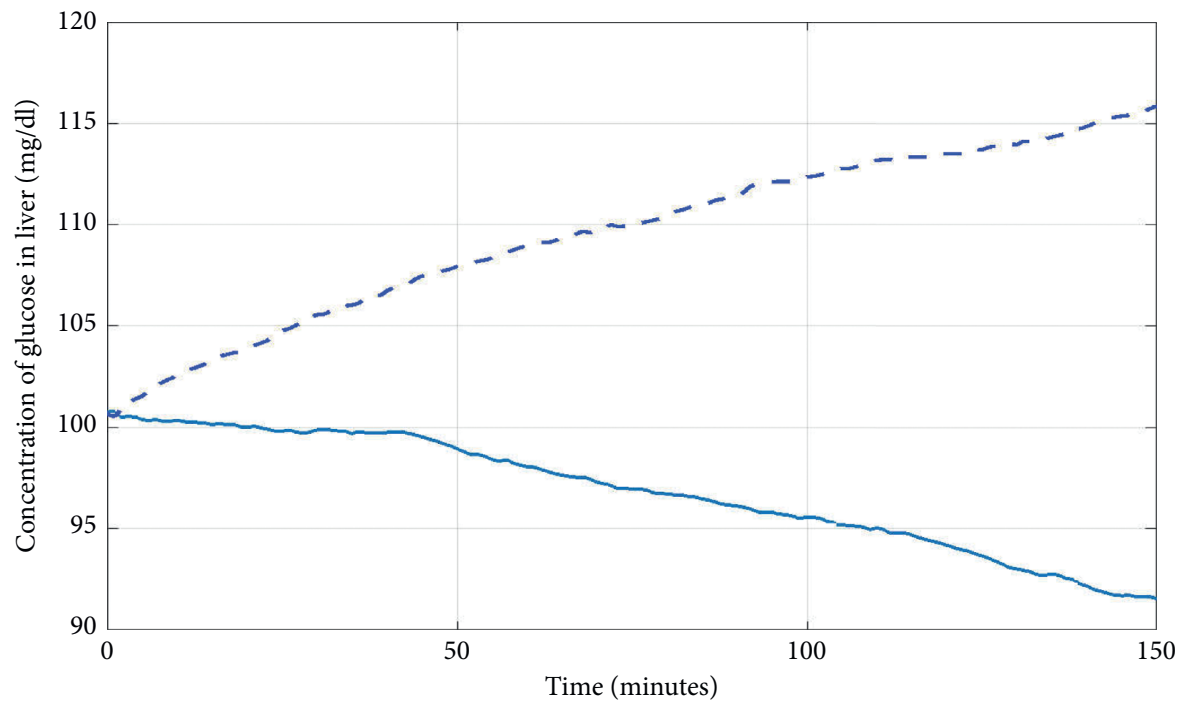

Figure 8: Hepatic glucose profile during euglycemic hyperinsulinemic clamp test. The dashed line shows the type 2 diabetic patients hyperglycemic clamp test, while the solid line shows the same for healthy subjects.

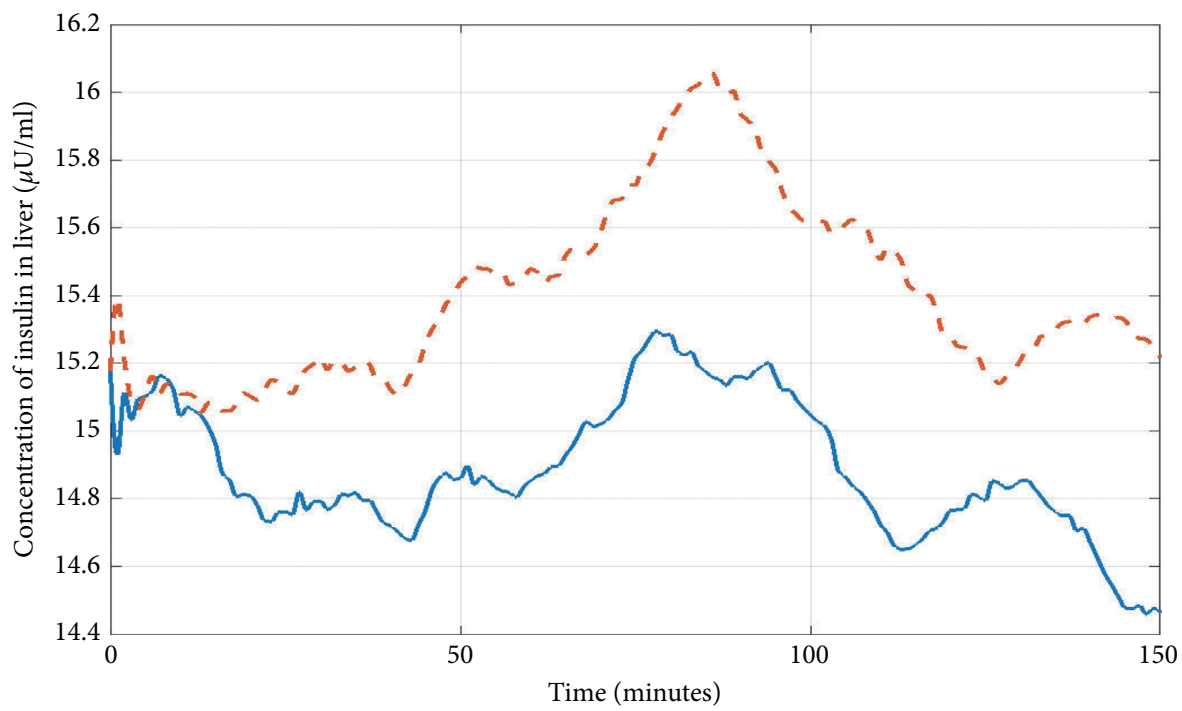

Figure 9: Hepatic insulin profile during euglycemic hyperinsulinemic clamp test. The dashed line shows the type 2 diabetic patients hyperglycemic clamp test, while the solid line shows the same for healthy subjects.

\section{Data Availability}

The description of how the data are found is presented in the manuscript.

\section{Conflicts of Interest}

The authors declare no conflicts of interest.

\section{Acknowledgments}

The authors would like to thank the study's subjects for their participation and acknowledge the assistance of medical doctors of public hospital in Kigali, Rwanda, with data collection and Ethical Committee of College of Medicine and Health Sciences (CMHS), Institutional Review Board (IRB), at the University of Rwanda for approving protocols. This work was supported by the University of Rwanda through its Directorate of Research and Innovation (Research Grant 2015/2016).

\section{References}

[1] ADA (American Diabetes Association), "Diagnosis and classification of diabetes mellitus," Diabetes Care, vol. 32, no. 1, pp. S62-S69, 2009.

[2] I. MacFarlane, M. Bliss, and J. Jackson, History of Diabetes, Textbook of Diabetes, 2nd edition, 1997. 
[3] G. Soltesz, C. Patterson, and G. Dahlquist, "Diabetes in the young: a global perspective," IDF Diabetes Atlas Fourth Edition, pp. 1-36, International Diabetes Federation, Brussels, Belgium, 2009.

[4] CDA (Canadian Diabetes Association). Clinical Practice Guidelines Expert Committee, "Definition, classification and diagnosis of diabetes and other dysglycemic categories," Canadian Journal of Diabetes, vol. 32, pp. S10-S13, 2008.

[5] G. Roglic, N. Unwin, P. H. Bennett et al., "The burden of mortality attributable to diabetes: realistic estimates for the year 2000," Diabetes Care, vol. 28, no. 9, pp. 2130-2135, 2008.

[6] International Diabetes Federation, United for Diabetes Campaign: Key Messages, International Diabetes Federation, Brussels, Belgium, 2007.

[7] L. Guariguata, D. R. Whiting, I. Hambleton, J. Beagley, U. Linnenkamp, and J. E. Shaw, "Global estimates of diabetes prevalence for 2013 and projections for 2035," Diabetes Research and Clinical Practice, vol. 103, no. 2, pp. 137-149, 2014.

[8] G. Roglic and N. Unwin, "Mortality attributable to diabetes: estimates for the year 2010," IDF Diabetes Atlas Fourth Edition, pp. 1-36, International Diabetes Federation, Brussels, Belgium, 2010.

[9] International Diabetes Federation, "IDF diabetes atlas, seventh edition," International Diabetes, vol. 64, no. 12 , pp. 3967-3977, 2016.

[10] E. H. Hilawe, H. Yatsuya, L. Kawaguchi, and A. Aoyama, "Differences by sex in the prevalence of diabetes mellitus, impaired fasting glycaemia and impaired glucose tolerance in sub-Saharan Africa: a systematic review and meta-analysis," Bulletin of the World Health Organization, vol. 91, no. 9, pp. 671D-682D, 2013.

[11] Republic of Rwanda Ministry of Health, Rwanda Noncommunicable Diseases Risk Factors Report, Republic of Rwanda Ministry of Health, Kigali, Rwanda, 2015.

[12] International Diabetes Federation, IDF Diabetes Atlas, Seventh Edition, the Country Report, 2015, http://reports. instantatlas.com/report/view/

846e76122b5f476fa6ef09471965aedd/RWA, International Diabetes Federation, Brussels, Belgium, February 2016, http:// reports.instantatlas.com/report/view/ 846e76122b5f476fa6ef09471965aedd/RWA.

[13] G. Bukhman and A. Kidder, The Partners in Health Guide to Chronic Care Integration for Endemic Non-communicable Diseases, Partners In Health, Boston, MA, USA, Rwanda edition, 2001.

[14] N. Tapela, H. Habineza, S. Anoke et al., "Diabetes in rural Rwanda: high retention and positive outcomes after 24 months of follow-up in the setting of chronic care integration," International Journal of Diabetes and Clinical Research, vol. 3, no. 2, pp. 1-6, 2016.

[15] E. B. Ketema and K. T. Kibret, "Correlation of fasting and postprandial plasma glucose with $\mathrm{HbA1c}$ in assessing glycemic control; systematic review and meta-analysis," Archives of Public Health, vol. 73, no. 1, p. 43, 2015.

[16] J. Kropff and J. H. DeVries, "Continuous glucose monitoring, future products, and update on worldwide artificial pancreas projects," Diabetes Technology and Therapeutics, vol. 18, no. 2, pp. 253-263, 2016.

[17] A. J. Young, H. Thabit, S. R. Heller et al., "Holistic impact of closed-loop technology on people with type 1 diabetes," Journal of Diabetes Science and Technology, vol. 9, no. 4, pp. 932-933, 2015.
[18] R. Atun and E. A. M. Gale, "The challenge of diabetes in SubSaharan Africa," The Lancet Diabetes \& Endocrinology, vol. 3, no. 9, pp. 675-677, 2015.

[19] G. Cedersund and P. Strålfors, "Putting the pieces together in diabetes research: towards a hierarchical model of whole-body glucose homeostasis," European Journal of Pharmaceutical Sciences, vol. 36, no. 1, pp. 91-104, 2009.

[20] A. Makroglou, J. Li, and Y. Kuang, "Mathematical models and software tools for the glucose-insulin regulatory system and diabetes: an overview," Applied Numerical Mathematics, vol. 56, no. 3-4, pp. 559-573, 2006.

[21] A. Mari, "Mathematical modeling in glucose metabolism and insulin secretion," Current Opinion in Clinical Nutrition and Metabolic Care, vol. 5, no. 5, pp. 495-501, 2000.

[22] H. Wang, J. Li, and Y. Kuang, "Mathematical modeling and qualitative analysis of insulin therapies," Mathematical Biosciences, vol. 210, no. 1, pp. 17-33, 2007.

[23] R. N. Bergman, L. S. Phillips, and C. Cobelli, "Physiologic evaluation of factors controlling glucose tolerance in man: measurement of insulin sensitivity and beta-cell glucose sensitivity from the response to intravenous glucose," Journal of Clinical Investigation, vol. 68, no. 6, pp. 1456-1467, 1981.

[24] L. Kovas, "Extension of the Bergman minimal model for the glucose-insulin interaction," Periodica Polytechnica Electrical Engineering, vol. 50, no. 1-2, pp. 23-32, 2006.

[25] C. Cobelli and A. Mari, "Validation of mathematical models of complex endocrine-metabolic systems. A case study on a model of glucose regulation," Medical \& Biological Engineering \& Computing, vol. 21, no. 4, pp. 390-399, 1983.

[26] J. Sorensen, A physiological model of glucose metabolism in man and its use to design and assess improved insulin therapies for diabetes, Ph.D. thesis, Massachusetts Institute of Technology, Cambridge, MA, USA, 1985.

[27] R. Hovorka, V. Canonico, L. J. Chassin et al., "Nonlinear model predictive control of glucose concentration in subjects with type 1 diabetes," Physiological Measurement, vol. 25, no. 4, pp. 905-920, 2004.

[28] O. Vahidi, Dynamic modeling of glucose metabolism for the assessment of type II diabetes mellitus, Ph.D. thesis, The University of British Columbia, Vancouver, Canada, 2015.

[29] J. M. Ntaganda, F. Minani, W. Banzi et al., "Simplified mathematical model of glucose-insulin system," American Journal of Computational Mathematics, vol. 8, no. 3, pp. 233-244, 2018.

[30] C. N. Eleni and A. W. Smyth, "The unscented Kalman filter and particle filter methods for nonlinear structural system identification with non-collocated heterogeneous sensing," Journal of Structural Control and Monitoring, vol. 16, no. 1, pp. 99-123, 2009.

[31] M. Wu and A. W. Smyth, "Application of the unscented Kalman filter for real-time nonlinear structural system identification," Structural Control and Health Monitoring, vol. 14, no. 7, pp. 971-990, 2007.

[32] L. H. Nguyen and N. A. Goulet, "Anomaly detection with the switching Kalman filter for structural health monitoring," Structural Control and Health Monitoring, vol. 25, no. 2, Article ID e2136, 2018.

[33] C. Andrieu, A. Doucet, S. S. Singh, and V. B. Tadic, "Particle methods for change detection, system identification, and control," Proceedings of the IEEE, vol. 92, no. 3, pp. 423-438, 2004.

[34] S. Arulampalam, S. Maskell, N. Gordon, and T. Clapp, “A tutorial on particle filters for on-line non-linear/non- 
Gaussian Bayesian tracking," IEEE Transactions on Signal Processing, vol. 50, no. 2, pp. 174-188, 2001.

[35] A. Doucet, S. Godsill, and C. Andrieu, "On sequential Monte Carlo sampling methods for Bayesian filtering," Statistics and Computing, vol. 10, no. 3, pp. 197-208, 2000.

[36] N. J. Gordon, D. J. Salmond, and A. F. M. Smith, "Novel approach to nonlinear/non-Gaussian Bayesian state estimation," IEE Proceedings F Radar and Signal Processing, vol. 140, no. 2, pp. 107-113, 1993.

[37] H. Landahl and G. Grodsky, "Comparison of models of insulin release," Bulletin of Mathematical Biology, vol. 44, no. 3, pp. 399-409, 1982.

[38] W. B. Rhoten, "Perifusion of saurian pancreatic islets and biphasic insulin release following glucose stimulation," Comparative Biochemistry and Physiology Part A: Physiology, vol. 45, no. 4, pp. 1001-1007, 1973.

[39] S. Nagasaka, K. Tokuyama, I. Kusaka et al., "Endogenous glucose production and glucose effectiveness in type 2 diabetic subjects derived from stable-labeled minimal model approach," Diabetes, vol. 48, no. 5, pp. 1054-1060, 1999.

[40] R. A. Defronzo, J. D. Tobin, and R. Andres, "Glucose clamp technique: a method for quantifying insulin secretion and resistance," American Journal of Physiology-Endocrinology and Metabolism, vol. 237, no. 3, pp. E214-E223, 1979. 